\title{
Endoscope-Assisted Minimally Invasive Microsurgical Removal of a Medium-Sized Acoustic Neuroma (T3a) through a Retrosigmoid Mini-Craniotomy with Preservation of Hearing
}

\author{
Peter Kurucz ${ }^{1,2}$ Laszlo Barany ${ }^{3} \quad$ Michael Buchfelder $^{2} \quad$ Oliver Ganslandt ${ }^{1}$ \\ ${ }^{1}$ Department of Neurosurgery, Klinikum Stuttgart, Stuttgart, \\ Germany \\ ${ }^{2}$ Department of Neurosurgery, Friedrich-Alexander-Universitat \\ Erlangen-Nurnberg, Erlangen, Bayern, Germany \\ ${ }^{3}$ Laboratory for Applied and Clinical Anatomy, Department of \\ Anatomy, Histology and Embryology, Semmelweis University, \\ Budapest, Hungary \\ J Neurol Surg B 2019;80(suppl S3):S276-S278. \\ Address for correspondence Peter Kurucz, MD, Department of \\ Neurosurgery, Klinikum Stuttgart, Kriegsbergstrasse 60, Stuttgart \\ 70174, Germany (e-mail: p.Kurucz@klinikum-stuttgart.de).
}

\begin{abstract}
Keywords

- acoustic neuroma

- endoscope-assisted microsurgery

- minimally invasive

- keyhole approach

- retrosigmoid craniotomy

A 45-year-old male patient with reduced hearing in the right ear was diagnosed with a medium-sized (T3a) acoustic neuroma. The operation was performed through an individually tailored retrosigmoid mini-craniotomy. Endoscope-assisted microsurgical technique was applied to early detect the location of the facial and cochlear nerves as well as to look around the hidden corners during the surgery. The entire operation was performed without using brain retractors. The moderate intrameatal extension of the tumor and the use of angled endoscopes allowed to avoid the drilling of the internal auditory meatus. The lesion could be completely removed and the patient showed a satisfactory hearing recovery in the follow-up examinations 3 months after the surgery. The link to the video can be found at: https://youtu.be/dAYLakih924.
\end{abstract}

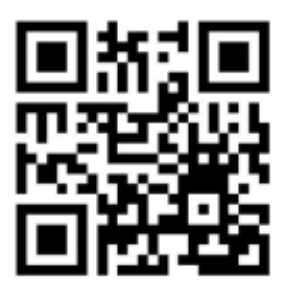

received

April 22, 2018 accepted after revision November 11, 2018 published online February 18, 2019 www.thieme.com/skullbasevideos

www.thieme.com/jnlsbvideos
License terms

c. $\oplus \triangleq \$$
DOI https://doi.org/

10.1055/s-0038-1677488. ISSN 2193-6331. (c) 2019 Georg Thieme Verlag KG
Stuttgart · New York \author{
.
}

(1) 

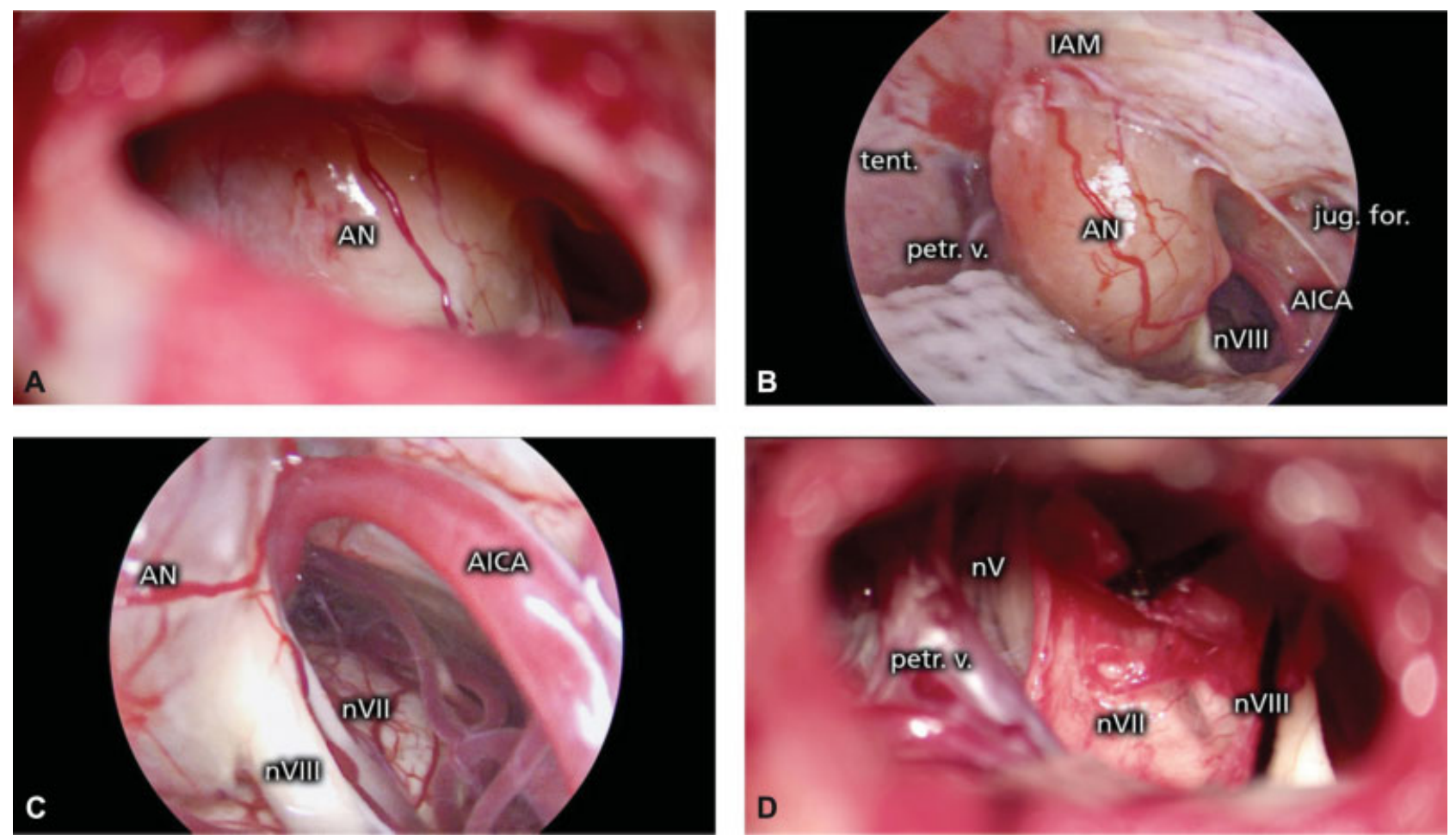

Fig. 1 Pre- and Postoperative MRI images. Preoperative CISS (A) and contrast-agent enhanced T1-weighted (B) MRI-scans showed a mediumsized (T3a) acoustic neuroma on the right side with slightly protrusion into the internal auditory canal. The cerebellopontine angle is filled by the tumor and the surface of the brainstem is in a gently contact with the lesion. The postoperative CISS (C) and contrast-agent enhanced T1weighted (D) MRI-scans 3 months after the surgery verified the complete tumor removal. 

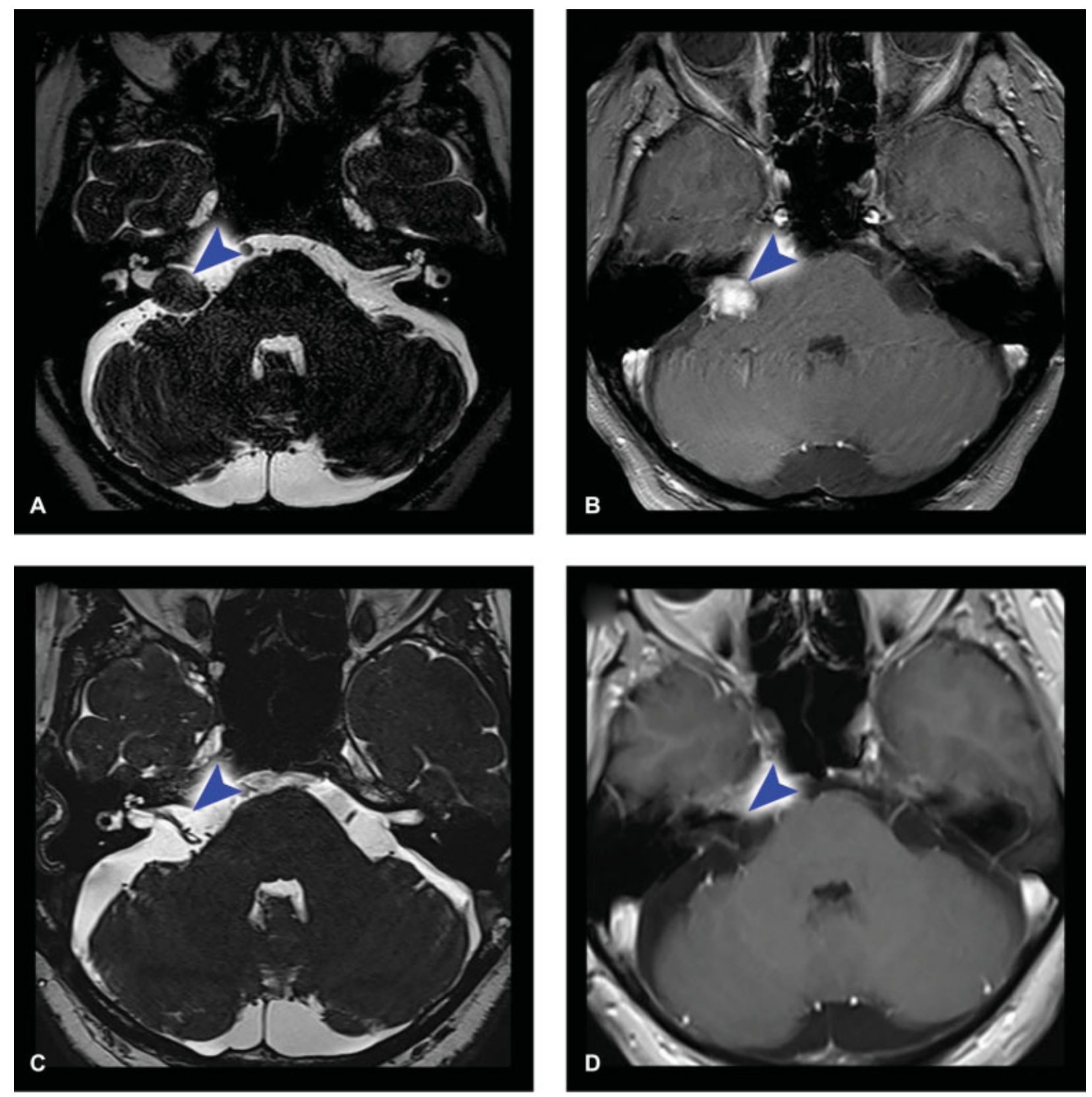

Fig. 2 Intraoperative photomicrographs. (A) Microscopic view of the tumor through the retrosigmoid mini-craniotomy. (B) Overview of the tumor and the surrounding anatomical structures through the 30 degree endoscope. (C) Detection of the cochlear and facial nerves with a 30 degree endoscope prior to the tumor resection. (D) Microscopic view of the operative field with the intact cochlear and facial nerves after complete resection of the tumor. AICA, anterior inferior cerebellar artery; AN, acoustic neuroma; IAM, internal auditory meatus; $\mathrm{nV}$, trigeminal nerve; nVII, facial nerve; nVIII, cochlear nerve; petr. v., superior petrosal vein; tent, tentorium.

Conflict of Interest

None declared. 